\title{
Shaping the future of the COVID-19 pandemic in Canada
}

\author{
Ashleigh R. Tuite PhD MPH, Amy L. Greer PhD MSc
}

Cite as: CMAJ 2020 September 14;192:E1074-5. doi: 10.1503/cmaj.201758; early-released August 10, 2020

See related article at www.cmaj.ca/lookup/doi/10.1503/cmaj.200990

C anada is in a period of easing public health measures after initiating an early and aggressive response to the coronavirus disease 2019 (COVID-19) pandemic. Many other countries are doing the same. Determining the best path forward now involves, in many ways, a much more difficult series of decisions than those taken in the initial phase of the pandemic. But this is the time to identify the future we want and work toward realizing that goal.

A related mathematical modelling study by $\mathrm{Ng}$ and colleagues ${ }^{1}$ examines potential future trajectories for the COVID-19 epidemic in the Canadian population under different nonpharmaceutical intervention scenarios. The findings show that our future is highly uncertain and dependent on our choices. With high levels of testing for severe acute respiratory syndrome coronavirus 2 (SARS-CoV-2) infection, timely contact tracing, rapid isolation, physical distancing and community closures, $\mathrm{Ng}$ and colleagues project negligible population attack rates $(<1 \%$ of the population infected); with erosion of these measures, however, up to $56 \%$ of Canada's population could acquire SARS-CoV-2 infection. Given such a wide range of possibilities, how do we ensure that we follow the path that limits virus transmission and prevents severe outcomes? And, given that SARS-CoV-2 will be circulating in the Canadian population for the foreseeable future, how can transmission be controlled in a sustainable way that limits serious societal and economic harms?

Mathematical modelling can help to guide policy-makers in working out what is required to achieve epidemic control. As Ng and colleagues show, ${ }^{1}$ combinations of interventions are likely to be more effective than single public health measures, particularly as communities move away from large-scale closures of schools and workplaces. But the effectiveness of different interventions for reducing transmission is only one piece of the puzzle. Public compliance with these measures is also an important consideration, particularly as the burden of infection is reduced, the threat begins to feel less urgent and "pandemic fatigue" sets in. In the absence of a vaccine or other therapeutic breakthrough, a long road lies ahead. Ng and colleagues' model has a

\section{KEY POINTS}

- The future of the coronavirus disease 2019 pandemic in Canada is uncertain and highly dependent on actions taken today.

- Multiple public health interventions will likely be required for the foreseeable future to prevent uncontrolled severe acute respiratory syndrome coronavirus 2 (SARS-CoV-2) transmission.

- Mathematical models can help in understanding what combinations of public health measures are needed for epidemic control as Canada eases restrictions, but determining how to reopen requires input from other sectors and will reveal our societal priorities and preferences.

- Although eliminating SARS-CoV-2 may not be achievable in the Canadian context, it is a worthwhile goal.

lengthy time horizon (January 2022), and even the limited scenarios that can lead to elimination of COVID-19 often achieve this only after they have been in place for more than a year. ${ }^{1}$

There is no single correct path forward in this pandemic. Policymakers need to determine how to strategically use public health measures to achieve improved epidemic control and determine whether there are aspects of the initial pandemic response that require additional investment and resources. Ultimately, there is no route that will not disadvantage some groups, which means choosing carefully which aspects of our society and economy are most important to us. Walsh proposed that policy-makers consider what aspects of society to reopen through the lens of a "social interaction budget."2 Each social interaction carries risk of SARS-CoV-2 transmission, which has been shown to be enhanced in crowded, close-contact, indoor settings. ${ }^{3}$ Only a finite budget of social interactions can be spent before COVID-19 transmission substantially increases. Exhausting that budget will necessitate reimplementation of aggressive lockdown measures. For instance, if reopening of schools and reuniting families and caregivers with long-term care residents is to be prioritized, we may have to forgo the reopening of indoor bars and restaurants, as concurrency of these activities is likely to substantially 
increase SARS-CoV-2 transmission. Similarly, making children's return to school as safe and inclusive as possible may require cancellation of higher-risk activities within schools, such as schoolwide assemblies and choir groups. ${ }^{4}$

Decisions like these are complex, expensive and fundamentally political. They will have far-reaching (and undoubtedly unanticipated) consequences. They have the potential to further enhance or assuage existing health and economic inequalities, ${ }^{5}$ and, furthermore, these decisions must be made under conditions of uncertainty. Models of transmission of SARS-CoV-2 can help make explicit some underlying assumptions and priorities, and allow decision-makers to hedge their bets based on the best available information. However, most models consider only health outcomes and rarely capture the indirect social and economic consequences associated with any given choice.

Canada's social interaction budget is not fixed, however; it can increase with more effective interventions. For instance, a recent mathematical modelling study showed the importance of widespread testing of symptomatic COVID-19 cases and rapid contact tracing. ${ }^{6}$ Investments in increased testing capacity and more rapid test turnaround times and initiation of contact tracing could allow for a larger social interaction budget. Other countries that have successfully controlled their COVID-19 epidemics can provide useful lessons. For example, Japan's cluster-busting approach, ${ }^{3}$ which focuses on rapidly finding clusters of cases and determining their common characteristics, could be used if widespread testing is deemed infeasible.

$\mathrm{Ng}$ and colleagues also show that achieving elimination of SARS-CoV-2 without restrictive measures is unlikely. ${ }^{1}$ The term "elimination" in public health refers to the reduction of incidence of infection caused by a specific pathogen to zero in a defined geographical region. ${ }^{7}$ As long as transmission occurs outside of the region that is aiming for elimination, risk of reintroduction remains. As such, the goal of elimination might be questioned in a country with high global connectivity like Canada. Although achieving elimination may not be feasible, we would argue that it is nonetheless a worthy goal to work toward. Elimination should be Canada's aim and focus. Through strong leadership, ingenuity, organization and solid public health practice, Canada will be able to find imported cases and limit community transmission, which will make the reopening of society and a return to a sense of normalcy more likely. Indeed, better control of COVID-19 epidemics seems to be the key to minimizing economic damage. ${ }^{8}$

Both SARS-CoV-2 and COVID-19 are understood far better now than when the virus was first identified. Certain public health interventions have been shown to effectively control transmission. The actions we take and the policies we implement now will shape the future of this pandemic.

\section{References}

1. Ng V, Fazil A, Waddell LA, et al. Projected effects of nonpharmaceutical public health interventions to prevent resurgence of SARS-CoV-2 transmission in Canada. CMAJ 2020 Aug 10 [Epub ahead of print]. doi: 10.1503/cmaj.200990.

2. Walsh $\mathrm{S}$. We are asking the wrong questions about easing lockdown [blog]. The BMJ Opinon 2020 June 2. London (UK): The BMJ. Available: https://blogs.bmj. $\mathrm{com} / \mathrm{bmj} / 2020 / 06 / 02 /$ sebastian-walsh-we-are-asking-the-wrong-questions-about -easing-lockdown/ (accessed 2020 July 17).

3. Furuse $\mathrm{Y}$, Sando $\mathrm{E}$, Tsuchiya N, et al. Clusters of coronavirus disease in communities, Japan, January-April 2020. Emerg Infect Dis 2020 June 10;26 [Epub ahead of print]. doi: 10.3201/eid2609.202272.

4. Hamner L, Dubbel P, Capron I, et al. High SARS-CoV-2 attack rate following exposure at a choir practice-Skagit County, Washington, March 2020. MMWR Morb Mortal Wkly Rep 2020;69:606-10.

5. Ali S, Asaria M, Stranges S. COVID-19 and inequality: Are we all in this together? Can J Public Health 2020;111:415-6.

6. Kucharski AJ, Klepac P, Conlan AJK, et al.; CMMID COVID-19 working group. Effectiveness of isolation, testing, contact tracing, and physical distancing on reducing transmission of SARS-CoV-2 in different settings: a mathematical modelling study. Lancet Infect Dis 2020 June 15 [Epub ahead of print]. doi: 10.1016/S1473-3099(20)30457-6

7. Dowdle WR. The principles of disease elimination and eradication. Bull World Health Organ 1998;76(Suppl 2):22-5.

8. Aum S, Lee SY, Shin Y. COVID-19 doesn't need lockdowns to destroy jobs: the effects of local outbreaks in Korea. SSRN 2020 June 3. Available: https://ssrn. com/abstract $=3615585$ (accessed 2020 July 17).

\section{Competing interests: None declared.}

This article was solicited and has not been peer reviewed.

Affiliations: Dalla Lana School of Public Health (Tuite), University of Toronto, Toronto, Ont.; Department of Population Medicine (Greer), University of Guelph, Guelph, Ont.

Contributors: Both authors contributed to the conception of the work, drafted the manuscript, revised it critically for important intellectual content, gave final approval of the version to be published and agreed to be accountable for all aspects of the work.

Correspondence to: Ashleigh Tuite, ashleigh.tuite@utoronto.ca 